\title{
Laser Direct Writing of Highly Conductive Circuits on Modified Polyimide
}

\author{
Tingxiu Hou ${ }^{1}$, Shi Bai ${ }^{1}$, Weiping Zhou ${ }^{1}$ and Anming $\mathrm{Hu}^{1,2}$ \\ ${ }^{1}$ Institute of Laser Engineering, Beijing University of Technology, Beijing100124, China \\ ${ }^{2}$ Department of Mechanical, Aerospace and Biomedical Engineering, University of Tennessee \\ Knoxville, Knoxville, Tennessee 37996, USA \\ E-mail: anminghu@bjut.edu.cn
}

\begin{abstract}
One-step laser direct writing (LDW) is becoming an effective method in the production of conductive circuits on the surface of polyimide (PI). The laser radiation can significantly improve the conductivity of the polymer and result in potential applications in the microelectronics and sensing. We studied the effect of laser power and scanning speed on the conductivity of the LDW generated circuit with assistance of scanning electron microscope, Raman spectroscopy and electrochemical workstation. Although laser direct writing technique can greatly improve the conductivity of polyimide films, there is a certain limitation of this improvement. Therefore, we adopted two methods to further enhance the electrical conductivity: (1) PI surface coated with metal ion solution to form the surface composite of metal and carbonated structure through laser irradiation; and (2) the addition of metal nano-plates and graphene oxide into poly (amic acid) (PAA) solution as the precursor of polyimide. The results show that the latter is more effective. The conductive circuit on polyimide generated by LDW can be used as flexible and wearable sensors to monitor the change of stress and as a platform for other sensing.
\end{abstract}

DOI: $10.2961 /$ jlmn.2017.01.0003

Keywords: laser direct writing, polyimide, carbonization, flexible stress sensors, laser processing

\section{Introduction}

With the development of portable microelectronics, electronic circuits are urgently required to be flexible and lightweight. Flexible electronic circuit has novel application in wearable electronic devices [1], flexible sensors [2, 3], and human-machine interaction [4]. Among many flexible substrates, polyimide (PI) is one of polymer materials with excellent mechanical and thermal performance. PI film with high insulation can be use at temperature range from $200{ }^{\circ} \mathrm{C}$ to $300{ }^{\circ} \mathrm{C}$ for a long time [5]. Due to their high thermal stability and superior mechanical strength, polyimides are usually used in aviation and aerospace field, information industry, and electrical engineering [6-8]. The "green" fabrication process of flexible electronic circuit seeks the objectives for pollution reduction, simplified operations, and products miniaturization. Comparing with conventional techniques, such as photolithography [9], screen printing [10] and stamp imprinting [11], laser direct writing (LDW) is a one-step processing method with higher repeatability and precision [12]. The discovery that the laser radiation leads to the increase of the conductivity of polymer surface is important for potential applications in microelectronics industry. The study of Schumann, Feurer, and Phillips et al showed that the electrical conductivity of PI can improve 15 16 orders of magnitude under the irradiation of ultraviolet laser [13-15]. Lin et al used a $\mathrm{CO}_{2}$ laser to irradiate PI film to generate graphene stacked structures [16]. Subsequently, researchers prepared supercapacitor energy storage devices and stretchable sensors by a $\mathrm{CO}_{2}$ laser LDW method $[17,18]$ and femtosecond laser LDW [3].

Although laser irradiation on PI surface can greatly improve its electrical conductivity, there is a certain gap between irradiated PI and a good conductor. Many researchers dedicated their efforts to the study of the interaction mechanism of laser and polymer materials in order to optimize the electrical conductivity of polymer materials. At present, the photo-thermal mechanism and photodegradation mechanism have been identified for relevant laser-induced carbonization [12, 19, 20]. To further elucidate these mechanisms we systematically investigated laser parameters for irradiation. Moreover, we adopted surface modification and inner modification to enhance the electrical conductivity.

In this paper, we firstly studied the effect of laser power and scanning speed on the conductivity of LDW generated conductive circuits. The $405 \mathrm{~nm}$ laser beam irradiates on the PI film surface to fabricate conductive circuits with the assistance of a 2D motorized platform. In order to further improve the electrical conductivity, we comapred three different methods. First, laser irradiated PI surface was coated with metal ion solution to form the surface composite of metal and carbonated structure. Second, silver nanoplates were added into poly (amic acid) (PAA) solution to achieve inner modification. Finally, graphene oxide/polyimide composite films were fabricated and laser reduced to improve the electrical conductivity. Our work has potential applications in high sensitive flexible and wearable sensors. 


\section{Experiment}

\subsection{Fabrication of flexible electronic circuit by laser direct writing method}

A laser direct writing system was equipped with a continuous laser with a wavelength of $405 \mathrm{~nm}$, as shown in Fig. 1. A beam expander and a focusing objective were used to focus the laser beam on the surface of polyimide (PI) substrates. The beam size of the $405 \mathrm{~nm}$ laser is 200um. The PI film with a thickness of $175 \mu \mathrm{m}$ was placed on a twodimensional mobile platform controlled by a computer. The PI film was purchased by Shenzhen Golden Leaves Electronic Material Limited Liability Company (Kapton $\left.{ }^{\circledR}\right)$. The laser power and scanning speed were varied to investigate their effects on the PI electrical property.

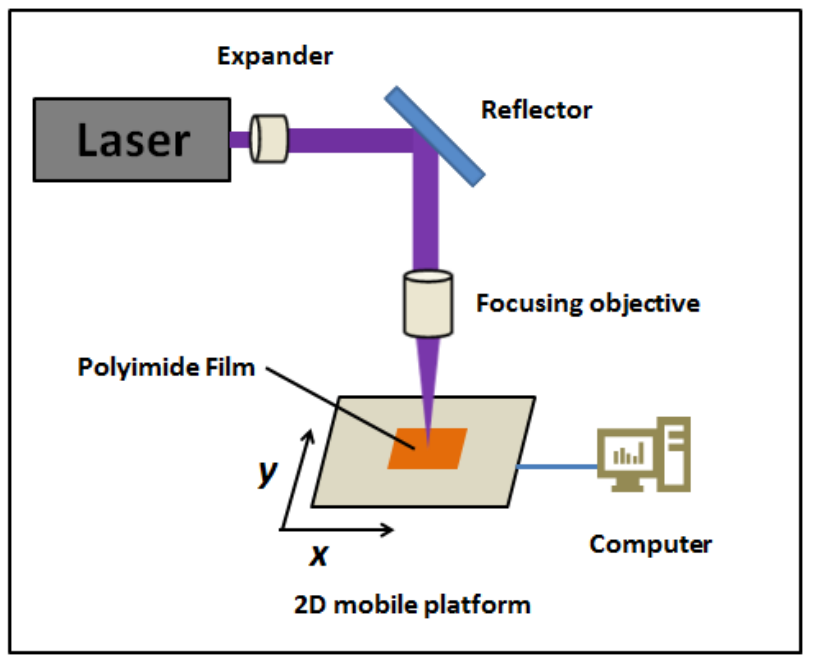

Fig. 1 Schematic of the laser direct writing system

\subsection{The preparation of modified polyimides}

Three solutions were instigated for modified polyimide films. Firstly, the PI surface was coated with silver ion solution. The synthesis method of silver ion solution was adopted from Maruo and Saeki [21, 22]. Silver nitrate, polyvinylpyrrolidone (PVP) (K90), and ethanol, all analytically pure, were purchased from Tianjin Fu Chen Chemical Reagent Factory. 0.25g PVP was dissolved in $10 \mathrm{ml}$ ethanol and $0.4 \mathrm{~g}$ silver nitrate was dissolved in $2 \mathrm{~mL}$ deionized water. The mixture of two kinds of solution was placed in dark room for 30 minutes. The PI film was immersed into alcohol to ultrasonic cleaning and then washed with deionized water. The PI film coated with the mixed solution was dried at $50{ }^{\circ} \mathrm{C}$ for $10 \mathrm{~min}$.

Secondly, we prepared the single-crystalline silver plates/polyimide composite film. The synthesis of singlecrystalline Ag plate was based on the method reported by Chunyuan Wang [23]. Polyvinylpyrrolidone (PVP) (K30), ethylene glycol (EG), ammonium hydroxide $\left(\mathrm{NH}_{4} \mathrm{OH}\right)$, hydrogen peroxide $\left(\mathrm{H}_{2} \mathrm{O}_{2}\right)$, and chloroplatinic acid $\left(\mathrm{H}_{2} \mathrm{PtCl}_{6}\right)$ were purchased from Tianjin $\mathrm{Fu}$ Chen Chemical Reagent Factory. $1.7328 \mathrm{~g} \mathrm{AgNO}_{3}$ dissolved into $60 \mathrm{ml}$ ethylene glycol solution (EG). Then, $4 \mathrm{ml} \mathrm{NH}_{4} \mathrm{OH}(15 \mathrm{M})$ was injected into solution. And 2 g PVP (K30) was added slowly to form uniform solution with the assist of magnetic stirring. $2.06 \mathrm{ml} \mathrm{H}_{2} \mathrm{PtCl}_{6} \cdot x \mathrm{H}_{2} \mathrm{O}(0.02 \mathrm{M})$ and $5.2 \mathrm{ml} \mathrm{H}_{2} \mathrm{O}_{2}$ (30\%) was injected successively into the mixture solution. After one day, single-crystalline silver plates washed by deionized water were was dried at $60{ }^{\circ} \mathrm{C}$ for $1 \mathrm{~h}$. $0.0628 \mathrm{~g}$ singlecrystalline silver plates dissolve again into $20 \mathrm{ml}$ dimethylacetamide and mixed with $80 \mathrm{ml}$ poly (amic acid) solution. Finally, the mixed viscous solution was coated on glass substrate with the assist of a coating machine. The single-crystalline silver plates/ polyimide composite film was formed after drying at $250^{\circ} \mathrm{C}$ for $1 \mathrm{~h}$.

Thirdly, laser assisted reduction of graphene oxide/polyimide composite film to improve the electrical conductivity. We prepared the single-crystalline silver plates/polyimide composite film. The synthesis method of graphene oxide (GO) was improved based on modified Hummer method [24, 25]. Graphite powder, KMnO4, $\mathrm{H}_{2} \mathrm{SO}_{4}$, and $\mathrm{H}_{3} \mathrm{PO}_{4}$ were purchased from Tianjin $\mathrm{Fu}$ Chen Chemical Reagent Factory. $1.5 \mathrm{~g}$ graphite powder and $9 \mathrm{~g}$ $\mathrm{KMnO} 4$ was added successively into a mixture of $200 \mathrm{~mL}$ $\mathrm{H}_{2} \mathrm{SO}_{4} / \mathrm{H}_{3} \mathrm{PO}_{4}$ (9:1). The mixture was stirred and heated to $\sim 55{ }^{\circ} \mathrm{C}$ for $12 \mathrm{~h}$. After cooling to room temperature, $200 \mathrm{ml}$ ice was added into the solution. Then $4 \mathrm{ml} \mathrm{H}_{2} \mathrm{O}_{2}$ (30\%) was injected slowly until the color of solution change to bright yellow. After stirring for $12 \mathrm{~h}$ and standing for $1 \mathrm{~h}$, the supernatant is removed. GO solution obtained after washed by using $10 \mathrm{wt} \% \mathrm{HCl}$ and deionized water. GO solution was dried at $60{ }^{\circ} \mathrm{C}$ for $2 \mathrm{~h}$ to obtain the GO powder. 0.1315 g GO powder dissolve again into $20 \mathrm{ml}$ dimethylacetamide and mixed with $80 \mathrm{ml}$ poly (amic acid) solution. Finally, the mixed viscous solution was coated on glass substrate with the assist of a coating machine. The $\mathrm{GO} /$ polyimide composite film was formed after drying at $250{ }^{\circ} \mathrm{C}$ for $1 \mathrm{~h}$.

\subsection{Instruments}

Morphology was investigated by field emission scanning electron microscopy (Hitachi S-4800, Japan) and atomic force microscopy (Bruker Multimode 8, United States). Raman spectra were acquired using a confocal microprobe Raman system (inVia-Reflex, Renishaw, United Kingdom) with an excitation laser wavelength of $532 \mathrm{~nm}$. Absorption spectrum was measured by a UV-Vis spectrophotometer (UV-9000S, Metash, China). The electrochemical performances were examined by using an electrochemical workstation (Chenhua CHI600E, China). Infrared spectrophotometer (TP270-30A, China) was used to analyze the structure of organic compounds.

\section{Results and discussions}

\subsection{Morphological characterization and electrical property of LDW generated conductive circuits}

Laser direct writing can generate conductive circuit due to the local carbonization of PI under laser irradiation through photo-thermal and photo-degradation mechanisms $[20,26]$. For the photo-thermal mechanism, PI absorbs the incident photon energy and converts it into heat. This induces extremely high temperature in the irradiated region and results in carbonization [19]. The transition of molecules of polyimide from the ground state to the excited state after absorb photon energy leading to chain breaks and the formation of free radical in photo-degradation mechanism. For the long-wavelength laser, the PI film absorbs strongly to cause the undesired effect, such as ablation and deformation. Therefore, we adopt laser with the 
wavelength of $405 \mathrm{~nm}$ to write electric patterns on the surface of polyimide films. To study the carbonization degree in the one-step LDW process, we varied the parameters of laser through controlling the laser power $(\mathrm{P})$ in a range of $1.5 \mathrm{~W}<\mathrm{P}<5.1 \mathrm{~W}$ and the laser scanning speed $(\mathrm{S})$ in a range of $0.03 \mathrm{~mm} / \mathrm{s}<\mathrm{S}<3 \mathrm{~mm} / \mathrm{s}$.

Fig. 2a and Fig. 2b are SEM images of the microscopic morphology of the irradiated regions. Foam-like hierarchical porous morphologies of the $1 \mathrm{~cm}$ conductive lines prepared by using LDW $(\mathrm{P}=5 \mathrm{~W}, \mathrm{~S}=2.86 \mathrm{~mm} / \mathrm{s})$ were observed. These pores with size of tens of microns are attributed to the rapid releasing of gases produced in the photo-thermal carbonization process of PI under the laser irradiation. A lot of smaller pores also appear in the carbonized skeletons.
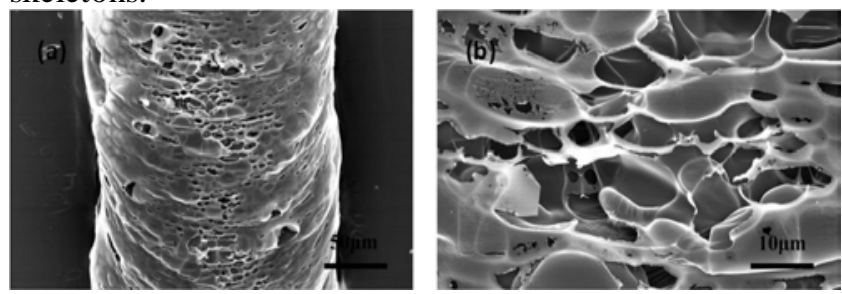

Fig.2 SEM images of laser irradiated $\mathrm{PIs}(\mathrm{P}=5 \mathrm{~W}$ and $\mathrm{S}=2.86$ $\mathrm{mm} / \mathrm{s}$ )

Fig. 3a, Fig. 3b and Fig. 3c show the line features created at different laser powers while maintaining a constant scanning speed. When the laser power is lower than $1.5 \mathrm{~W}$, it could not form a continuous pattern. While PI film would occur deformation and carbonized line fall off at excessively laser powers higher than 5W. Different scanning speed matched appropriate power range. When laser scanning speed were $0.03 \mathrm{~mm} / \mathrm{s}, 0.10 \mathrm{~mm} / \mathrm{s}$ and $0.17 \mathrm{~mm} / \mathrm{s}$, the range of laser power respectively matched with $1.6 \mathrm{~W}<\mathrm{P}$ $<2.9 \mathrm{~W}, 2.4 \mathrm{~W}<\mathrm{P}<4.7 \mathrm{~W}$ and $3.1 \mathrm{~W}<\mathrm{P}<5.1 \mathrm{~W}$. Within the intermediate laser powers, the width of line increases at a higher power accordingly. It can vary from $\sim 148$ um to $\sim 289.5 \mathrm{~mm}$ at $\mathrm{S}=0.03 \mathrm{~mm} / \mathrm{s}$ (from $\sim 110.5 \mathrm{um}$ to $\sim 252.5$ $\mathrm{mm}$ at $\mathrm{S}=0.10 \mathrm{~mm} / \mathrm{s}$ and from $\sim 96 \mathrm{um}$ to $\sim 236.5 \mathrm{~mm}$ at $\mathrm{S}=0.17 \mathrm{~mm} / \mathrm{s}$ ). The height of conductive line generated by LDW gradually increases with the increase of laser power. It can vary from $\sim 30$ um to $\sim 112 \mathrm{~mm}$ at $\mathrm{S}=0.03 \mathrm{~mm} / \mathrm{s}$ (from $\sim 21$ um to $\sim 99 \mathrm{~mm}$ at $\mathrm{S}=0.10 \mathrm{~mm} / \mathrm{s}$ and from $\sim 12$ um to $\sim 66 \mathrm{~mm}$ at $\mathrm{S}=0.17 \mathrm{~mm} / \mathrm{s}$ ). The electrical conductivity of the electrical line was also studied by an electrochemical workstation. The conductivity of carbonize line of PI film can reach to $6.6,7.8$ and $9.2 \mathrm{~S} / \mathrm{cm}$ at $\mathrm{S}=0.03 \mathrm{~mm} / \mathrm{s}$, $\mathrm{S}=0.10 \mathrm{~mm} / \mathrm{s}$ and $\mathrm{S}=0.17$. These values are slightly higher than femtosecond laser irradiated PIs [17]. Therefore, appropriately choosing appropriate laser powers and scanning speeds can optimize the electrical conductivity of laser carbonization line on PI film surface.

Raman spectra peaks of irradiated PIs can be found at $1360 \mathrm{~cm}^{-1}$ (D-band) and1580 $\mathrm{cm}^{-1}$ (G-band), as shown in Fig. 3d. The G-band is the primary phonon arising from lattice stretching in the $\mathrm{C}-\mathrm{C}$ bonding in the graphitic plane. The D-band corresponds to the defects in the graphitic lattice. The ratio of the peak intensity $\left(\mathrm{I}_{\mathrm{D}} / \mathrm{I}_{\mathrm{G}}\right)$ of the $\mathrm{D}$ and $\mathrm{G}$ bands reflecting defects in the graphitic material reduced from 0.91 to 0.71 with the increase of laser power. These values are also lower than femtosecond laser irradiated PIs [17] and laser reduced graphene oxide indicating a lower defected structure [27].
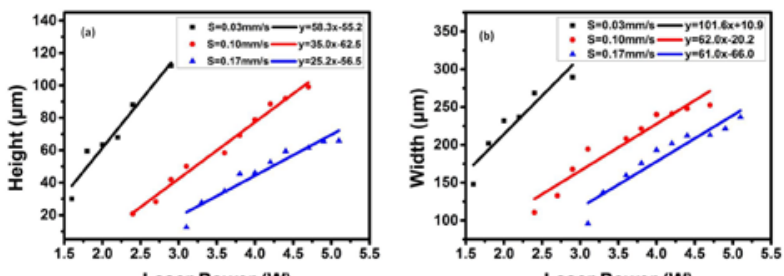

Laser Power (W)
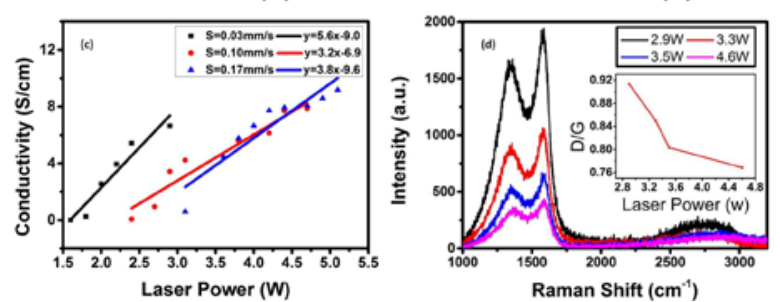

Fig. 3 Laser power effect and scanning speed on (a) line width, (b) height, (c) conductivity and (d) Raman spectra of the LDW generated electronic circuit at $S=2.86 \mathrm{~m} / \mathrm{s}$.

\subsection{Properties of single-crystalline silver plates and grapheme oxide}

In order to further improve the conductivity of the modified PI, we choose single-crystalline silver plates and graphene oxide (GO) added to polyimide.

As shown in Fig. 4a, most of single-crystalline silver nano-plates appear as regular hexagons. The size of Ag nano-plates ranges between $14.42 \mu \mathrm{m}$ and $38.76 \mu \mathrm{m}$ and the mean size is $\sim 24.1 \mu \mathrm{m}$ (Fig. 4b). High-resolution scanning electron microscopy images (Fig. 4c and Fig. 4d) clearly show that these crystals have $\sim 500 \mathrm{~nm}$ thickness. Only the Ag (111) peak was observed in the XRD data. Comparing with previous works, the narrow full-width at half maximum (FWHM) $\left(\sim 0.13^{\circ}\right)$ suggests that the crystallinity of grown crystals is greatly improved [28, 29]. The surface roughness of single-crystalline silver nano-plates is $<1 \mathrm{~nm}$ over a large area $\left(1 \mu \mathrm{m}^{2}\right)$ as measured by atomic force microscopy (Fig. 4e and Fig. 4f).
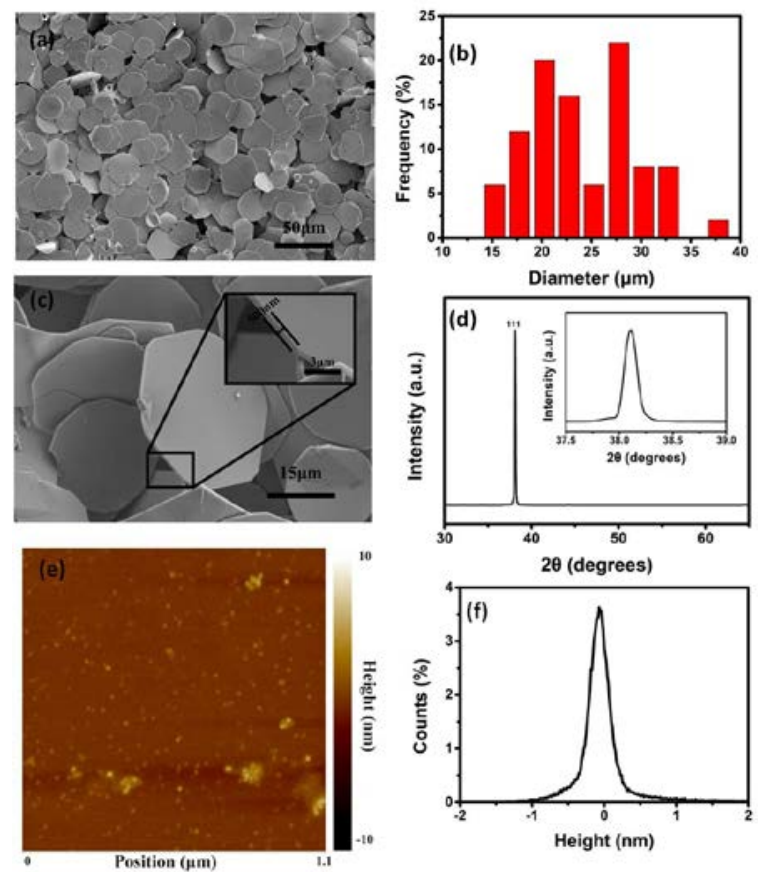

Fig. 4 Structural properties of single-crystalline silver plates: (a, c) SEM images, (b) the statistic size distribution of Ag plates, (d) XRD wide-scan patterns, (e) a typical AFM image and (f) the distribution of surface height over a $1 \mu \mathrm{m}^{2}$ 
The synthetized GO is dispersed into water to form a pale yellow homogeneous colloidal solution. It is clear that the GO shows a crumpled morphology, as shown in Fig. 5a. The average thickness of GO, measured from the height profile of the AFM image (Fig. 5b), is about $1.5 \mathrm{~nm}$. In the Fig. 5c, we can distinguish a characteristic absorption peak at about $230 \mathrm{~nm}$ that shows $\pi-\pi^{*}$ transitions of $\mathrm{C}=\mathrm{C}$ bonds. There is a shoulder at around $300 \mathrm{~nm}$ in absorption spectrum of the GO solution, corresponding to the $n-\pi *$ transitions of the $\mathrm{C}=\mathrm{O}$ bond in $s p^{3}$ hybrid regions [30]. IR spectra presented in Fig. 5d show characteristic $\mathrm{OH}\left(3390 \mathrm{~cm}^{-1}\right)$, $\mathrm{C}=\mathrm{O}\left(1740 \mathrm{~cm}^{-1}\right), \mathrm{COOH}\left(1619 \mathrm{~cm}^{-1}\right)$, aromatic $\mathrm{C}=\mathrm{C}(1500$ $\left.\mathrm{cm}^{-1}\right)$ and $\mathrm{C}-\mathrm{O}\left(1400 \mathrm{~cm}^{-1}\right)$ signals of synthesized GO [31].
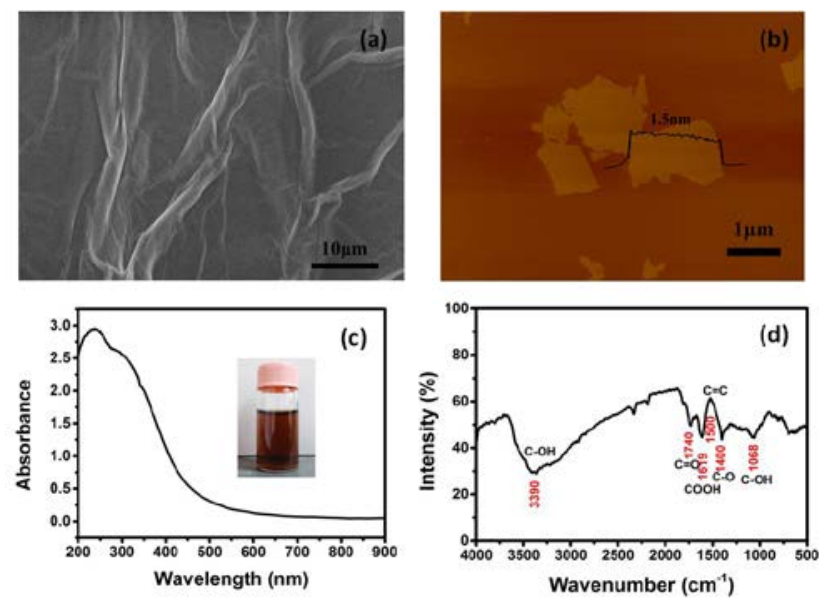

Fig. 5 Representatives (a) SEM image, (b) AFM image, (c) UV-Vis-near infrared spectroscopy and (d) infrared spectrum of graphene oxide

\subsection{The improvement of electrical conductivity of mod- ified PI films}

Poly (amic acid) solution, the precursor of polyimide, was coated on glass substrate and then dried at $60{ }^{\circ} \mathrm{C}$ for 1 $\mathrm{h}$ to form PAA film. For the IR spectra of PAA film with a $20 \mu \mathrm{m}$ thickness, the peak at $3265 \mathrm{~cm}^{-1}$ reflects the existence of -NH-, and $1663 \mathrm{~cm}^{-1}, 1542 \mathrm{~cm}^{-1}$ and $1237 \mathrm{~cm}^{-1}$ are characteristic peaks of amide groups. PAA solution dehydrates to form polyimide films, when heated for $30 \mathrm{~min}$ at $300{ }^{\circ} \mathrm{C}$. The typical peaks of PI films appear at $1773 \mathrm{~cm}^{-1}$ and $1719 \mathrm{~cm}^{-1}$ (Carbonyl), $1500 \mathrm{~cm}^{-1}$ (Aromatic C-C), $1377 \mathrm{~cm}^{-1}$ (Imide C-N), $1242 \mathrm{~cm}^{-1}$ (C-O-C), $880 \mathrm{~cm}^{-1}$ and $823 \mathrm{~cm}^{-1}$ (Aromatic C-H bending), and $723 \mathrm{~cm}-1$ (Imide C$\mathrm{N}$ or $\mathrm{C}-\mathrm{H}$ bending), as shown in Fig. 6b. The IR spectra of the PI film are consistent with the commercial Kapton polyimide, as shown in Fig. 6c.

We study the conductivity of the LDW generated electronic line on modified PI films. When the laser scanning speed is $0.03 \mathrm{~mm} / \mathrm{s}$, the maximum conductivity is respectively $132 \mathrm{~S} / \mathrm{cm}(\mathrm{P}=1.1 \mathrm{~W}), 27 \mathrm{~S} / \mathrm{cm}(\mathrm{P}=3.9 \mathrm{~W})$ and 37 $\mathrm{S} / \mathrm{cm}(\mathrm{P}=5 \mathrm{~W})$ for PI coated with $\mathrm{Ag}$ ion solution, Ag nano-plates/polyimide composite film and GO/polyimide composite film, as shown in Fig. 7.

When laser irradiates the PI coated with Ag ion solution, photo-reduction and carbonization will occur at the same time [19, 27]. Silver nanoparticles through photoreduction attach on the carbonized skeleton at low laser power. With the increase of laser power, the size of silver nanoparticles decreases probably due to the effect of laser ablation. At a scan speed of $\mathrm{S}=0.03 \mathrm{~mm} / \mathrm{s}$ and laser power of $\mathrm{P}=1.1 \mathrm{~W}$, the conductivity of LDW on PI coated with $\mathrm{Ag}$ ion solution increases 13 times than laser irradiation PI directly. From EDS spectra, we can also confirm that silver nanoparticles attac skeleton of carbonization.

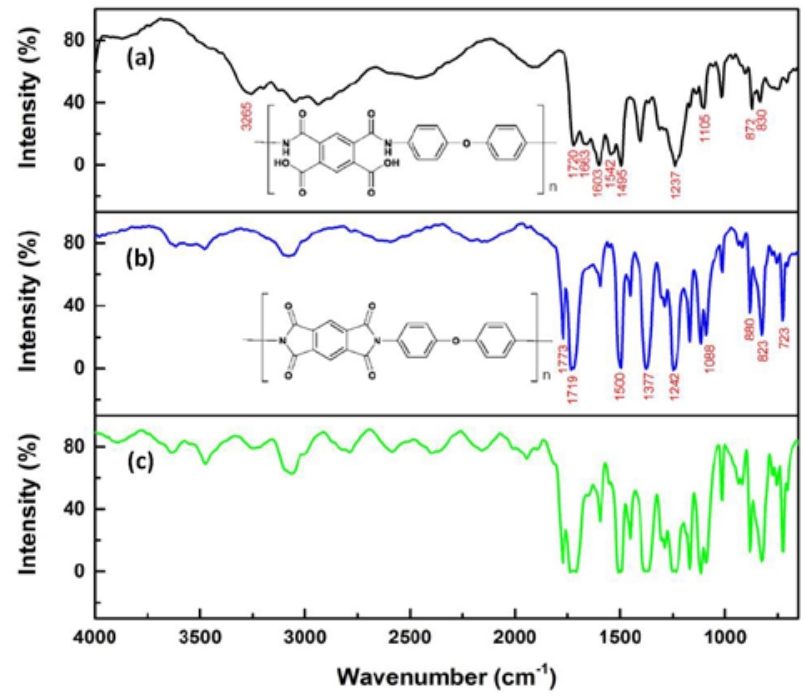

Fig. 6 Infrared spectra of (a) a PAA film, (b) a PI film and (c) a commercial Kapton film.

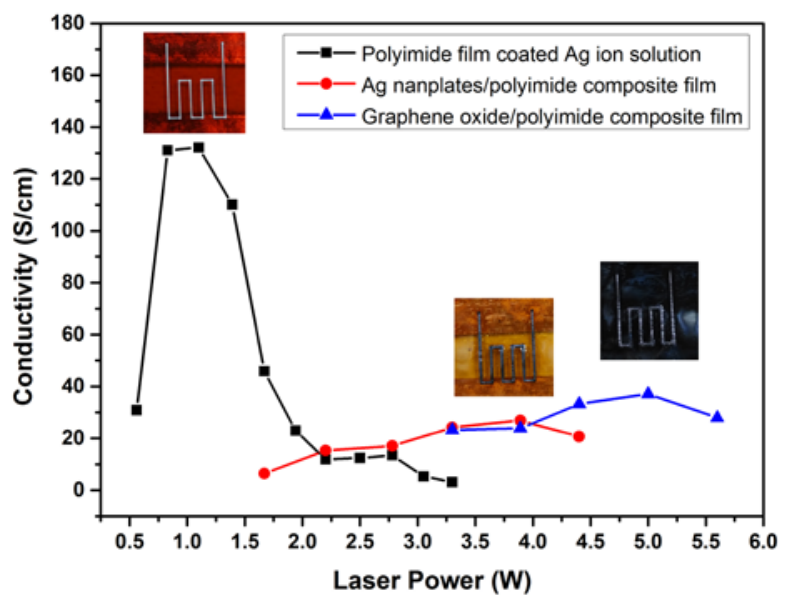

Fig. 7 Conductivity of LDW generated electronic line on three kinds of modified PI films.
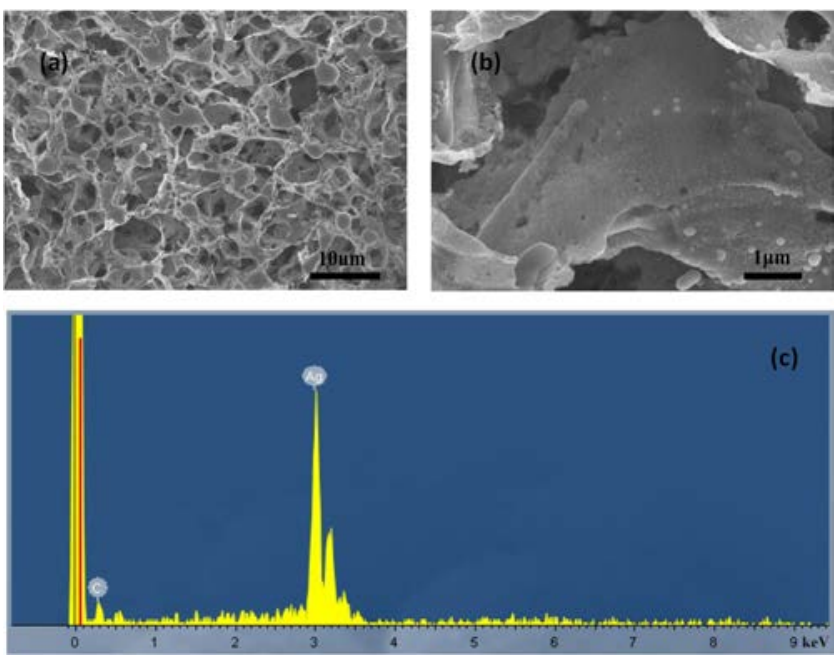

Fig. 8 (a), (b) SEM images and (c) EDS spectrum of composite structure of porous carbon and silver nanoparticles. 
At $\mathrm{S}=0.03 \mathrm{~mm} / \mathrm{s}$ and $\mathrm{P}=1.7 \mathrm{~W}$, single-crystalline silver nano-plates are dispersed in hollow filamentary structures due to low carbonization, as shown in Fig. 9a and 9b. As the laser power increases to $3.9 \mathrm{~W}$, silver nano-plate can be fused with porous carbon matrix with the assistance of laser melting, as shown in Fig. 9c and 9d. At $S=0.03 \mathrm{~mm} / \mathrm{s}$ and $\mathrm{P}=3.9 \mathrm{~W}$, the conductivity of LDW on Ag nanoplates/polyimide composite film only increased 2 times than that of directly irradiated PI without adding silver nanoplates.

The conductivity of electronic line generated by LDW on $\mathrm{GO} /$ polyimide composite film increases 3 times at $\mathrm{P}=5$ $\mathrm{W}$ and $\mathrm{S}=0.03 \mathrm{~mm} / \mathrm{s}$. Under laser irradiation, reductions of GO and carbonization occur at the same time. The reduced graphene oxide (r-GO) is electrically conducting due to the removal of oxygen-containing functional groups of insulative GO by laser irradiation. Carbonated structures of polyimide combining with conductive r-GO improve electrical conductivity.

Comparing with three modification techniques, the conductivity of laser directly written PI coated with Ag ion solution would be improved in an order of magnitude. This arises from abundant silver nanoparticles through photoreduction attached on the porous carbonated structures. On the contrary, the binding force of large-size silver nanoplates and porous carbonated structure is weak. Parital reduction of graphene oxide can yield a limited improvement of the conductivity on GO/polyimide composite film. The uniform mixture of Ag nanoplates and GO is difficult to achieve in preparation of Ag nano-plates/polyimide composite films. Therefore, laser direct writing of PI coated with Ag ion solution is an optimum method with simple operation and high efficiency to improve the conductivity.
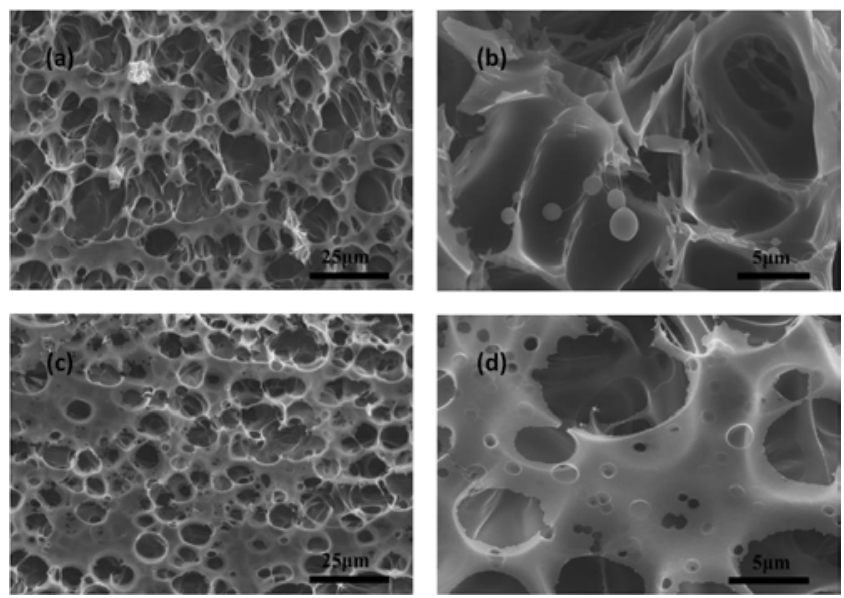

Fig. 9 SEM images of LDW generated electronic line on Ag nanoplate-doped PI with $\mathrm{S}=0.03 \mathrm{~mm} / \mathrm{s}$ at different laser power: (a) and (b) $\mathrm{P}=1.7 \mathrm{~W}$; (c) and (d) $\mathrm{P}=3.9 \mathrm{~W}$

\section{Conclusions}

In summary, direct writing with a wavelength of 405 $\mathrm{nm}$ laser has been used for preparation of electronic circuits through the carbonization of polyimide. The effect of laser power and scanning speed on the morphology and conductivity was systematically studied. Under the appropriate condition of laser irradiation, the conductivity of $9.2 \mathrm{~S} / \mathrm{cm}$ can be achieved. In order to further improve the conductivity, we adopted surface modification and inner modification to improve the performance of the polyimide film. Silver nanoparticles through photo-reduction attached on the skeleton of carbonization could increase the conductivity in an order of magnitude. The conductivity would increase 2 times with silver nano-plates-carbon composite. Laser reduction graphene oxide can also increase the conductivity. Laser direct writing of PI coated with Ag ion solution is thus a simple and efficient method to develop high conductive flexible circuits for wearable sensors.

\section{Acknowledgments}

This work is supported by Nature Science Foundation of China (NSFC) under grant number 51575016, a strategic project of Beijing Municipal Commission of Education (KZ20141000500, B-type), the special grant for the advanced laser technology group of Beijing and the oversea high-level talent project of Beijing.

\section{References}

[1] D. Kim, N. Lu, R. Ma, Y. Kim and R. Kim et al: Science, 333 (2011) 838.

[2] T. Sekitani, T. Yokota, U. Zschieschang, H. Klauk, S. Bauer, K. Takeuchi, M. Takamiya, T. Sakurai and T. Someya: Science, 326 (2009) 1516.

[3] C. Cheng, S. T. Wang, J. Wu, Y. C. Yu, R. Z. Li, S. Eda, J. G. Chen, G. Y. Feng, B. Lawrie and A. M. Hu: ACS Appl. Mater. Inter., 8 (2016) 17784.

[4] R. Z. Li, A. M. Hu, T. Zhang and K. D. Oakes: ACS. Appl. Mater. Inter., 56 (2014) 21721.

[5] S. H. Hsiao and Y. T. Chou: Polymer, 55 (2014) 2411.

[6] T. Kurosawa, T. Hiqashihara, and M. Ueda: Polym. Chem., 4 (2013) 16.

[7] J. Morikawa and T. Hashimoto: J. Appl.Phys., 105 (2009) 113506.

[8] X. Ji, Z. Wang, J. Yan, and Z. wang: Polymer, 74 (2015) 38.

[9] O. Catanescu and L. Chien: Adv. Mater., 17 (2005) 305.

[10] S. G. Im, P. J. Yoo, P. T. Hammond and K. K. Gleason: Adv. Mater., 19 (2007) 2863.

[11] J. H. Lim and C. A. Mirkin: Adv. Mater., 14 (2002) 1474.

[12]W. P. Zhou, S. Bai, Y. Ma, D. L. Ma, T. X. Hou, X. M. Shi and A. M. Hu: ACS Appl. Mater. Inter., (2016) DOI: 10.1021/acsami.6b07696.

[13] M. Schumann, R. Sauerbrey, and M. C. Smayling: Appl. Phys. Lett., 58 (1991) 428.

[14]T. Feurer, R. Sauerbrey, M. C. Smayling, et al: Appl. Phys. A, 56 (1993) 275.

[15] H. M. Phillips, S. Wahl, and R. Sauerbrey: Appl. Phys. Lett., 62 (1993) 2572.

[16] J. Lin, Z. Peng, Y. Liu, F. Ruiz-Zepeda, R. Ye, E. L. G. Samuel, M. J. Yacaman, B. I. Yakobson and J. M. Tour: Nat. Commun., 5 (2014) 5714.

[17] J. B. In, B. Hsia, J. Yoo, S. Hyun, C. Carraro, R. Maboudian and C. P. Grigoropoulos: Carbon, 83 (2015) 144.

[18]R. Rahimi, M. Ochoa, W. Yu, B. Ziaie: ACS Appl. Mater. Interfaces, 7 (2015) 4463.

[19] C. Zheng, A. M. Hu, K. D. Kihm, Q. Ma, R. Z. Li, T. Chen, and W. W. Duley: Small, 11 (2015) 3007. 
[20] A. M. Hu, M. Rybachuk, Q. B. Lu and W. W. Duley: Appl. Phys. Lett. 91 (2007) 131906.

[21] M. Shoji and S. Tatsuya: Opt. Express, 16 (2008) 1174.

[22] S. Bai, Y. H. Lin, X. P. Zhang, W. P. Zhou, T. Chen, Y. Ma, T. X. Hou, D. Bridges, K. D. Oakes and A. M. Hu: Plasmonics, 10 (2015) 1675.

[23] C. Y. Wang, H. Y. Chen, L. Y. Sun, W. L. Chen, Y. M. Chang, H. Ahn, X. Q. Li and S. Gwo: Nat. Commun., 6 (2015) 7734.

[24]W. S. Hummers and R. E. Offeman: J. Am. Chem. Soc., 80 (1958) 1339.

[25] H. A. Becerril, J. Mao, Z. F. Liu, R. M. Stoltenberg, Z. N. Bao, and Y. S. Chen: ACS Nano., 2 (2008) 463.

[26] R. Srinivasan, B. Braren, R. W. Dreyfus: J. Appl. Phy., 61 (1987) 372.
[27] R. Z. Li, R. Peng, K. D. Kihm, S. Bai, D. Bridges, U. Tumuluri, Z. Wu, T. Zhang, G. Compagnini, Z. Feng and A. M. Hu: Energy Environ. Sci., 9 (2016) 1458.

[28] J. H. Park, P. Ambwani, M. Manno, N. C. Lindquist, P. Nagpal, S. H. Oh, C. Leiqhton and D. J. Norris: Adv. Mater., 24 (2012) 3988.

[29] C. W. Chang, F. C. Lin, C. Y. Chiu, C. Y. Su, J. S. Huang, T. P. Perng and T. J. Yen: ACS Appl. Mater. Interfaces, 6 (2014) 11791.

[30] J. Shang, L. Ma, J. Li, W. Ai, T. Yu, G. G. Gurzadyan: Sci. Rep-UK, 2 (2012) 792.

[31] C. Gong, M. Acik. R. M. Abolfath, Y. Chabal and K. Cho: J. Phys. Chem. C, 116 (2012) 9969.

(Received: May 21, 2016, Accepted: November 23, 2016) 